\title{
Meeting report New tricks for an old-favorite model
}

\author{
Charalampos Rallis
}

Address: University College London, Department of Genetics, Evolution \& Environment and UCL Cancer Institute, Darwin Building, Gower Street, London WC1E 6BT, UK. Email: c.rallis@ucl.ac.uk

\begin{abstract}
A report of the 24th International Conference on Yeast Genetics and Molecular Biology, Manchester, UK, 19-24 July 2009.
\end{abstract}

The international yeast meetings are highly interactive conferences attracting scientists from diverse disciplines of fungal research. The 24th yeast meeting held in the University of Manchester presented recent advances ranging from basic cell biology to the use of yeast for industrial purposes and translational research. Here, I summarize a few highlights related to systems and synthetic biology, yeasts as model organisms in gene expression, aging and human disease studies and the use of yeast cells as factories.

\section{Yeast as model organism for aging studies and human disease}

Dennis Thiele (Durham University, North Carolina, USA) uses Saccharomyces cerevisiae as a model organism for studying metal metabolism. Iron is an essential micronutrient for almost all living organisms, serving as a cofactor for a huge variety of cellular processes. Iron imbalance is the primary nutritional disorder in the world (affecting around 2 billion people); however, cellular responses to iron deprivation are poorly understood. In response to iron deficiency, $S$. cerevisiae Cth2, an RNAbinding protein conserved in plants and mammals, is upregulated 200-fold. Cth2 binds to specific AU-rich elements in the 3' untranslated region of mRNAs and causes their degradation. Interestingly, degradation takes place in cytoplasmic processing bodies. Localization of Cth2 is a dynamic process: Cth2 travels to the nucleus before transcripts are delivered to processing bodies, and it interacts with target transcripts at or soon after their transcription. Microarray analysis for cells grown in low iron supply (simulating iron deficiency) have revealed mRNAs downregulated by Cth2, showing a global metabolic reprogramming driven by a posttranscriptional regulatory process controlled by iron deficiency.

Target of rapamycin (TOR) is the central component of a conserved eukaryotic signaling pathway that regulates cell and organismal growth in response to nutrient status. Growth rate correlates with ribosome number and metabolism, and TOR-dependent growth control in yeast and
Drosophila involves transcriptional regulation of ribosomal and metabolic genes. Suppression of the Drosophila TOR pathway results in prolonged pre-adult development and reduced larval and adult body sizes. TOR is linked to aging from yeast to mammals and deregulation of the pathway causes disease in humans (such as cancer, cardiac hypertrophy and inflammatory diseases). Mike Hall (Biozentrum, University of Basel, Switzerland) discussed recent findings of his group regarding the cross-talk between TOR with the protein kinase A (PKA) signaling pathway, which controls many similar processes. They established a pathway that includes a series of kinases, such as Sch9, in which TOR is upstream of PKA. Nevertheless, there are PKA targets that are TOR independent. Analysis showed that TOR is upstream of about half of the targets of the PKA pathway.

Replicative lifespan refers to the number of daughter cells a mother gives rise to before senescence. $S$. cerevisiae cells have limited replicative lifespan and show similar declining survival curves to those of metazoan cells. Longevity has a heritable basis, and epigenetic changes through the sirtuins, a group of deacetylases, have an important role in lifespan regulation. Sir2 promotes increased replicative lifespan, but the molecular basis of its action is poorly understood. Shelley Berger (The Wistar Institute, Philadelphia, USA) focused her talk on yeast Sir2, which removes lysine 16 from histone $\mathrm{H} 4$, promoting chromatin silencing. Old cells have decreased levels of Sir2 protein and of histone $\mathrm{H}_{4}$ lysine 56 acetylation, and $\mathrm{H}_{4}$ lysine 16 acetylation is increased. Sir2 antagonizes the action of Sas2 (a histone acetyltransferase) in subtelomeric regions, and telomeric silencing is reduced in old cells. This mechanism could represent an evolutionarily conserved function of sirtuins in regulation of replicative aging by maintenance of intact telomeric chromatin.

\section{Systems and synthetic biology}

Whole-genome duplication during evolution is common and provides novel genetic material as a major source of innovation and evolution. Nevertheless, it has been calculated that in fungi around $85 \%$ of duplicated genes have been lost. Evidence shows that genes encoding transcription factors and signal transducers are the best survivors following duplication. The reasons for this could 
be subfunctionalization (division of the original gene's functions between the duplicates), neofunctionalization (gain of new functions by duplicates), advantages gained in dosage balance in protein complexes or the need for increased dosage. Greg Amoutzias (University of Gent and Flanders Interuniversity Institute for Biotechnology (VIB), Gent, Belgium) presented a careful approach based on bioinformatics and a large phosphoproteomics dataset. The ancestral phosphorylation is linked to gene retention in future lineages. Statistical analysis showed that up to $7 \%$ of duplicated genes have been subfunctionalized. Reciprocal loss of ancestral phosphorylation sites and the gain of new ones are major contributors in the retention of the two surviving duplicates and in their acquisition of distinct functions. Increasing the number of phosphorylation sites increases the probabilities of both subfunctionalization and survival in future generations. Duplicated genes and their protein products are under tight regulatory control for example, by the proteasome.

Protein interactions underpin the genetic landscape. Kinases and phosphatases dynamically regulate most cellular processes, but because of the weak and transient interactions taking place, a kinase interactome has not been defined. Mike Tyers (University of Edinburgh, UK) presented a newly developed screen for weak interactions in budding yeast of all kinases and most of the phosphatases using multiple tags and nanoscale liquid chromatography-mass spectrometry. Low-confidence interactions were removed by a novel statistical algorithm that acts as a filter for large mass spectrometry datasets. His group uncovered 1,844 statistically significant interactions for 118 kinases, with more than $70 \%$ of them being confirmed using co-immunoprecipitation. Tyers provided examples of novel connections between signaling pathways, transcription-associated kinases, novel function of uncharacterized cell cycle checkpoint kinases and new effector kinases for the nutrient-responsive TOR signaling pathway. It was apparent that the kinome interlinks the proteome and that cellular behavior may be coordinated by a global signaling architecture.

Yitzak Pilpel (Weizmann Institute of Science, Rehovot, Israel) discussed adaptive environmental conditioning in microorganisms, a situation similar to Pavlovian neuronal/ cognitive conditioning. Escherichia coli encounters various metabolic environments in the digestive tract. The energy source early in the digestive tract is lactose, leading to activation of lactose operons, whereas later it is changed to maltose, which activates a different set of genes (maltose operons). Pilpel's group found that maltose operons are conditioned by lactose so that an intermediate level of maltose gene expression is achieved by lactose, whereas the converse situation is never observed. E. coli cells primed with lactose survive better in maltose than those that have not experienced lactose, and the expression of maltose genes is costly to the organism. These results show that $E$. coli tries to predict the change in the environment and programs genetic circuits for a better response and an increased chance of survival. Pilpel then moved to $S$. cerevisiae, where he showed that similar conditioned responses take place: oxidative protective genes are induced following mild heat shock (a cross-protection mechanism), but they are needed only in oxidative stress conditions, as shown by the fact that deletion mutants show the same survival as wild-type cells under heat shock.

Fritz Roth (Harvard University, Cambridge, USA) presented recent advances from his laboratory using parallel high-throughput sequencing (Solexa) and barcode fusion genetics for mass characterization of $S$. cerevisiae double mutants in competitive grown pool assays (a technique called BFG-GI). The technique involves the construction of double mutants, as for the traditional synthetic genetic array (SGA) approach, which are brought together in a huge pool. Cells are suspended in water-in-oil emulsion and are thus encapsulated within single droplets so that the barcode fusion PCR that follows binds together barcodes only from the same cell. Following emulsion purification and Solexa sequencing, Roth's group obtained 1.5 million mapped fused barcodes. Future efforts will also focus on adapting the system for protein interaction assays.

\section{Yeasts as cell factories}

Diethard Mattanovich (Universität für Bodencultur, Vienna, Austria) introduced the concept of systems biotechnology (applied research and development of microorganisms) and described the use of the methylotrophic yeast Pichia pastoris in metabolic engineering for the production of proteins (human antibodies) by secretion. The P. pastoris genome is fully sequenced and available through an open access genome browser [http://www.pichiagenome.org], DNA microarrays and a proteomics platform have been developed and metabolic modeling and flux analysis are currently being carried out. The Mattanovich group showed that temperature has a great effect on the secretion of heterologous proteins: a decrease in temperature results in an increase in secretion. Proteome analysis revealed that amino acid metabolism and ribosome biogenesis are elevated whereas chaperones and TCA (tricarboxylic acid) cycle enzymes are decreased. Transcriptome analysis showed that genes associated with translation and ribosome biogenesis are increased but stress response and TCA cycle genes are downregulated. Metabolomics analysis indicates that carbon metabolism is pushed towards biomass production instead of energy production. Overall, the results indicate that increased secretion could be a positive sideeffect of lowered energy demands. Mattanovich also presented screens for the generation of improved production strains. In one of these screens six novel secretion helper factors were identified, all of them cytosolic and endoplasmic reticulum chaperones. 
Strain engineering is used in industrial biotechnology for the generation of sustainable technologies in the production of fuels, chemicals and materials. $S$. cerevisiae is tolerant of ethanol and low $\mathrm{pH}$, is prone to contamination and shows robust aerobic and anaerobic growth. In order to achieve methods that make efficient use of resources without creating excess waste (eco-efficient methods), pentose sugars (such as xylose) need to be used as they are cheap, widely available in nature and can be obtained from plant and waste sources. However, S. cerevisiae cannot use pentoses. Genomic resources from a variety of microorganisms and systems biology combined with mutagenesis have been used to engineer yeasts for effective pentose fermentation. Merja Penttilä (VTT Technical Research Centre of Finland, Helsinki, Finland) presented the tremendous efforts being made towards alternative sugar utilization from yeast. $S$. cerevisiae strains that express xylose pathway genes from Pichia stipitis are making pentose fermentation to ethanol a reality. In addition, unconventional yeasts (such as Pichia, Candida and Hansenula) are attractive production hosts owing to their broader substrate range and tolerance to high temperatures and low $\mathrm{pH}$. Overall, this research will result in consolidated and cost-effective processes that will efficiently compete with petrochemistry.

\section{Control of gene expression}

Frank Holstege (University Medical Center, Utrecht, The Netherlands) described the efforts of his group towards the generation of genome control maps in $S$. cerevisiae. These are exhaustive wiring diagrams that describe in detail the contribution of regulatory factors for the regulation of every single gene. In order to increase the throughput of the mass microarray analysis used, they have robotically automated their procedures. Using external control calibration standards to assess improvements, the accuracy, precision and reproducibility of the microarray technology have greatly been improved. He presented examples of results following the expression profiling of 800 components of the regulatory machinery, such as the discovery of protein complexes that were also predicted from mass spectrometry data and an improved understanding of signaling pathways from uncovering the profiles for kinases and phosphatases and the complexity of the ubiquitin machinery.

Brenda Andrews (University of Toronto, Canada) introduced a fluorescent reporter SGA (R-SGA) experimental approach in which a promoter-green fluorescent protein (GFP) reporter along with a control promoter-red fluorescent protein (RFP) have been systematically introduced into SGAs. Fluorescence intensities from each reporter are assayed from individual colonies using a scanning fluorimager, and the ratio between GFP and RFP intensity reveals double mutants that show differential GFP expression. The system has been successfully used for studies on cell-cycle regulation of transcription in $S$. cerevisiae (GFP was driven by a cell-cycle-regulated promoter). The study has revealed a new regulatory pathway for controlling cell-cycle-dependent gene expression through the repression of histone transcription. The histone chaperone Rtt106 in combination with other two chaperones, Asfi and the HIR complex, create a repressive chromatin structure in the histone $\mathrm{H} 2 \mathrm{~A}$ promoter. The histone acetyltransferase Rtt109 relieves this repression, and the bromodomain-containing protein Yta7, which binds acetylated histones, is required for proper localization of Rtt106 to the promoter and prevents it from localizing to coding regions. This model was proposed as the primary mechanism of cell-cycle regulation of histone promoters.

Overall, the meeting reflected the divergent and highly interactive and vibrant yeast community. The 26th International Conference on Yeast Genetics and Molecular Biology takes place in Poland on 11-16 July 2011.

\section{Acknowledgements}

I thank Singer Instruments Co. Ltd for providing funds towards attending the meeting.

Published: 1 September 2009

doi:10.1186/gb-2009-10-9-315

(C) 2009 BioMed Central Ltd 